\title{
Consumo alimentar entre crianças brasileiras de dois a cinco anos de idade: Pesquisa Nacional de Demografia e Saúde (PNDS), 2006
}

\author{
Food intake among Brazilian children aged two to five years old: \\ National Survey on Demography and Health (NSDH), 2006
}

Mabel Nilson Alves ${ }^{1}$

Ludmila Correa Muniz ${ }^{1}$

Maria de Fátima Alves Vieira ${ }^{1}$

${ }^{1}$ Departamento de Nutrição, Universidade Federal de

Pelotas. R. Gomes Carneiro

1, Porto. 96.010-610

Pelotas RS Brasil.

mabelnalves@yahoo.com.br
Abstract The scope of this study was to describe aspects of the food intake of Brazilian children and its association with social and demographic factors. The 2006 food intake data of the National Survey on Demography and Health for women and children were analyzed. The analysis considered the complexity of the sample and included 3,083 children aged two to five years old. The outcome was evaluated by considering food intake markers. The estimates of these markers was described taking into account the children's gender, age and neighborhood as well as their mothers' age and education. Chi-square heterogeneity and linear tendency tests were used in the statistical analyses. It was seen that during the seven days before the interview $50 \%$ and $25.9 \%$ of the children evaluated, respectively, had not eaten greens and vegetables. In at least one day during the previous week the prevalence of consumption of fried food was of approximately 60\% and for soda and artificial juice was of $82 \%$. Female children whose mothers were older and had more education presented the highest prevalence in consumption of healthy food. As a result of these data, it is possible to say that the intensification of campaigns of nutritional education with innovatory approaches might help improve the food intake of children.

Key words Food consumption, Preschool, Child nutrition, NSDH
Resumo O objetivo deste estudo foi descrever características do consumo alimentar de crianças brasileiras e sua associação com fatores sociodemográficos. Foram analisados os dados de consumo alimentar da Pesquisa Nacional de Demografia e Saúde da Criança e da Mulher realizada em 2006. A análise considerou a complexidade amostral e incluiu 3.083 crianças de dois a cinco anos de idade. O desfecho foi avaliado a partir de marcadores alimentares. As estimativas desses marcadores foram descritas segundo sexo e idade da criança, local de residência, idade e escolaridade materna. Nas análises estatísticas foram utilizados testes qui-quadrado de heterogeneidade e de tendência linear. Verificou-se que 50\% e 25,9\% das crianças avaliadas não haviam consumido verduras de folhas e legumes nos sete dias anteriores ao da entrevista, respectivamente. A prevalência de consumo de pelo menos um dia na última semana de frituras foi cerca de $60 \%$ e de refrigerantes e sucos artificiais foi de $82 \%$. Crianças do sexo feminino, cujas mães possuíam maior idade e escolaridade, apresentaram as maiores prevalências de consumo de alimentos marcadores de alimentação saudável. Diante desses dados, a intensificação de campanhas de educação nutricional com abordagens inovadoras pode ajudar a melhorar a alimentação das crianças.

Palavras-chave Consumo alimentar, Pré-escolar, Nutrição infantil, PNDS 


\section{Introdução}

O grau de atendimento das necessidades básicas de alimentação, saneamento, acesso aos serviços de saúde, nível de renda e educação, entre outros, determinam as condições de vida e saúde de uma população ${ }^{1}$. Em crianças de dois a cinco anos de idade, a ingestão dietética está relacionada diretamente ao seu crescimento e desenvolvimento e também à formação de seus hábitos alimentares ${ }^{2,3}$.

Quando a alimentação é insuficiente pode levar à carência de micronutrientes causando imaturação biológica, em especial dos sistemas nervoso e imune, influenciando negativamente na eficácia de intervenções terapêuticas e propiciando infecções frequentes ${ }^{4,5}$. Além disso, um baixo aporte energético pode levar à desnutrição infantil que está fortemente associada com altura menor do adulto, menor escolaridade, além de produtividade e capital humano reduzidos 6 . Por outro lado, a ingestão dietética em excesso está associada à ocorrência, a médio e longo prazo, de doenças crônicas não transmissíveis como obesidade, dislipidemia, hipertensão arterial, entre outras?

Nas áreas metropolitanas do Brasil, no período de 1974 a 2003, o consumo alimentar da população caracterizou-se por teor excessivo de açúcar na dieta, aumento no aporte de gorduras em geral e de gorduras saturadas e consumo insuficiente de frutas e hortaliças ${ }^{8}$. Apesar de esses dados representarem a população adulta em geral, é possível inferir que as crianças estivessem seguindo o mesmo padrão de consumo alimentar de seus familiares, visto que se encontravam inseridas naquele contexto 2 . De acordo com a Pesquisa de Orçamentos Familiares (POF), as consequências desse perfil de consumo alimentar podem ter refletido na mudança observada no estado nutricional das crianças brasileiras menores de cinco de idade. Isto porque, no mesmo período, houve declínio da desnutrição e aumento das prevalências de sobrepeso e obesidade? 9 .

A partir do conhecimento do consumo alimentar de crianças, é possível planejar ações capazes de promover mudanças positivas no comportamento alimentar ainda na infância, fase na qual os hábitos de consumo estão sendo adquiridos e consolidados. Dessa forma, evitam-se agravos à saúde decorrentes de uma alimentação inadequada ${ }^{10}$. Embora a avaliação do consumo alimentar de crianças seja importante, existem poucos estudos de base populacional no país com essa abordagem.

Neste contexto, este trabalho teve por objetivo descrever o consumo alimentar de crianças de dois a cinco anos de idade, avaliadas na Pesquisa Nacional de Demografia e Saúde da Criança e da Mulher (PNDS), realizada em 2006.

\section{Métodos}

\section{População em estudo e amostragem}

Este estudo representa um recorte da PNDS 2006, na qual foram entrevistadas 15.575 mulheres de 15 a 49 anos de idade, residentes nos domicílios selecionados. Essas mulheres responderam a um questionário fornecendo informações socioeconômicas, demográficas, comportamentais e de saúde. Além disso, a saúde e a nutrição de seus filhos menores de cinco anos (nascidos a partir de janeiro de 2001) também foram investigadas. Neste trabalho, foram incluídas na análise apenas as crianças de dois a cinco anos de idade, tendo em vista que os resultados sobre consumo alimentar das crianças menores de dois anos já foram apresentados previamente ${ }^{11}$. O presente estudo é do tipo transversal, domiciliar por amostragem probabilística complexa, com representatividade nacional. As unidades amostrais foram selecionadas em dois estágios, onde as primárias eram compostas pelos setores censitários, e as secundárias, formadas pelos domicílios. Informações adicionais sobre o processo de amostragem estão detalhadamente descritas em outra publicaçãa ${ }^{11}$.

\section{Coleta de dados}

As informações foram coletadas por meio de questionário constituído pela Ficha do Domicílio e Questionário da Mulher. O questionário manteve a estrutura tradicional das outras edições da PNDS e as questões necessárias para comparabilidade nacional e internacional.

O questionário utilizado para avaliar o consumo alimentar das crianças era composto por uma lista de vinte alimentos, grupos de alimentos ou preparações: arroz ou macarrão; pão; feijão; batata ou mandioquinha (batata-baroa) ou mandioca (macaxeira, aipim) ou cará ou inhame ou abóbora (jerimum); verduras de folhas; legumes (tomate, cenoura, beterraba, berinjela, chuchu, abobrinha); frutas; carne de boi ou porco; fígado; frango; peixe; ovos; frituras; doces; biscoitos ou bolachas; salgadinhos caseiros (excluindo coxinha, kibe e outras frituras); salgadinhos em pacote; iogurte; refrigerantes e sucos artificiais; e suco natural. A frequência de consu- 
mo era referente aos sete dias anteriores ao da entrevista e as opções de resposta para cada item alimentar eram: não consumiu, consumiu um dia, dois a três dias, quatro a seis dias, todos os dias e não sabe ${ }^{11}$.

\section{Definição do desfecho e variáveis independentes}

O consumo alimentar das crianças de dois a cinco anos de idade constituiu o desfecho do presente estudo. Dentre os vinte itens alimentares anteriormente listados, dez (feijão, verduras de folhas, legumes, frutas, suco natural, frituras, doces, biscoitos ou bolachas, salgadinhos em pacote, refrigerantes ou sucos artificiais) foram utilizados nas análises. Os cinco primeiros foram considerados alimentos marcadores de alimentação saudável e os cinco últimos, de não saudável. Esta classificação foi baseada nas recomendações nutricionais para prevenção de doenças crônicas não transmissíveis e, também, em evidências que apontam a associação do consumo destes alimentos com o excesso de peso e outros agravos crônicos, como as dislipidemias ${ }^{12-14}$.

O consumo desses alimentos foi apresentado de duas formas: (1) através da distribuição da frequência semanal de consumo de cada item alimentar; (2) através da proporção de crianças que os consumiam de forma frequente (em pelo menos quatro dos sete dias que antecederam a entrevista) e menos frequente (entre zero e três dias dos sete dias que antecederam o estudo). Esta classificação da frequência de consumo consiste em uma adaptação da metodologia utilizada por Levy et al. ${ }^{15}$, que considerou consumo frequente aquele maior ou igual a cinco dias por semana. As opções de resposta eram fechadas no questionário da PNDS, então para este estudo, consumo frequente foi considerado de quatro a sete dias por semana e consumo não frequente de zero a três dias por semana.

Além disso, foram coletadas variáveis sociodemográficas, como idade da criança (obtida em meses e categorizada em 24-35; 36-47 e 48-59 meses), sexo da criança (masculino, feminino), macrorregião administrativa (Norte, Nordeste, Sudeste, Sul e Centro-Oeste), zona de moradia (urbana, rural), escolaridade materna (obtida em anos completos de estudo e categorizada em 0-4; 5-8; 9-11 e 12 ou mais anos de estudo) e idade materna (obtida em anos completos e agrupada em $<20,20-29,30-39,40$ anos ou mais). No presente estudo, a variável escolaridade materna foi utilizada como marcador de nível econômico.

\section{Digitação e análise dos dados}

O programa utilizado para a entrada de dados foi o CSPro (Census and Survey Processing System), software desenvolvido pelo Bureau do Censo Norte-Americano. Posteriormente, os dados foram transferidos e analisados no programa estatístico Stata versão 11.0, utilizando-se o comando svyset para definir os pesos amostrais e conglomerados e o prefixo svy para todas as análises realizadas, tendo em vista a complexidade do processo de amostragem.

Realizou-se análise descritiva das variáveis estudadas através de frequências relativas e absolutas. Foram realizados teste qui-quadrado de heterogeneidade e teste de Wald para tendência linear para avaliar as diferenças entre os grupos. Assumiu-se um nível de significância de 5\% (valor $\mathrm{p}<0,005)$.

\section{Aspectos éticos}

A PNDS-2006 recebeu a aprovação do Conselho de Ética em Pesquisa (CEP) do Centro de Referência e Treinamento DST/AIDS da Secretaria de Estado da Saúde (SP). Todas as pessoas que aceitaram participar do estudo assinaram um Termo de Consentimento Livre e Esclarecido.

\section{Resultados}

Nos 13.056 domicílios visitados, foram identificadas 3.083 crianças com dois a cinco anos de idade. As características dessas crianças e de suas mães são apresentadas na Tabela 1. Em cada um dos três grupos etários analisados encontravamse aproximadamente um terço das crianças. A maioria $(52,3 \%)$ era do sexo masculino, $41,0 \%$ residiam na região Sudeste e $79,7 \%$ na zona urbana. Em relação às mães, $57,4 \%$ possuíam entre 20 e 29 anos de idade e $78,9 \%$ tinham cinco ou mais anos completos de estudo.

A Tabela 2 apresenta a distribuição da frequência semanal de consumo dos alimentos marcadores de alimentação saudável e não saudável entre as crianças avaliadas. Quando analisados os alimentos marcadores de alimentação saudável, observou-se que a proporção de crianças que os consumiam de forma frequente variou de $20 \%$ a $76 \%$, sendo maior a proporção daquelas que consumiam feijão e frutas e menor daquelas que consumiam verduras de folhas e legumes. Verificou-se ainda, que cerca de 50\% e 25,9\% das crianças não haviam consumido verduras de folhas e 
Tabela 1. Distribuição da amostra segundo características sociodemográficas. Pesquisa Nacional de Demografia e Saúde (PNDS), 2006. $(\mathrm{n}=3.083)$.

\begin{tabular}{lcc}
\hline \multicolumn{1}{c}{ Variável } & $\mathbf{N}$ & $\%$ \\
\hline Idade da criança (meses) & & \\
$24-35$ & 1083 & 32,8 \\
$36-47$ & 1046 & 34,2 \\
$48-59$ & 1024 & 33,0 \\
Sexo & & \\
$\quad$ Masculino & 1590 & 52,3 \\
Feminino & 1493 & 47,7 \\
Macrorregião & & \\
$\quad$ Norte & 652 & 10,2 \\
$\quad$ Nordeste & 590 & 27,9 \\
$\quad$ Sudeste & 615 & 41,0 \\
$\quad$ Sul & 602 & 13,3 \\
$\quad$ Centro-oeste & 624 & 7,6 \\
Zona de moradia & & \\
$\quad$ Urbana & 1987 & 79,7 \\
$\quad$ Rural & 1096 & 20,3 \\
Idade materna (anos) & & \\
$\quad<20$ & 138 & 5,4 \\
$\quad$ 20 - 29 & 1772 & 57,4 \\
30 - 39 & 961 & 30,4 \\
40 - 49 & 212 & 6,8 \\
Escolaridade materna (anos) & & \\
$\quad$ 0 - 4 & 746 & 21,1 \\
5 - 8 & 936 & 37,0 \\
$\quad$ - 11 & 761 & 35,1 \\
$\quad$ ou mais & 151 & 6,8 \\
\hline
\end{tabular}

Percentual máximo de observações desconhecidas: 15,9\% $(\mathrm{n}=489)$ para a variável escolaridade materna. legumes nos sete dias anteriores ao da entrevista, respectivamente.

Com relação aos alimentos marcadores de alimentação não saudável (Tabela 2), observouse que a proporção de crianças que os consumiam de forma frequente variou de $13,5 \%$ a $65,4 \%$, sendo maior a proporção daquelas que consumiam biscoitos ou bolachas e menor daquelas que consumiam frituras. Foram registradas, ainda, altas proporções de crianças que haviam consumido alimentos desse grupo pelo menos um dia na última semana: de cerca de $60 \%$ (para frituras) a $82 \%$ (para refrigerantes e sucos artificiais).

O consumo frequente de alimentos marcadores de alimentação saudável segundo variáveis sociodemográficas está apresentado na Tabela 3. Uma maior prevalência de consumo frequente de feijão foi observada entre as crianças residentes na região Centro-oeste e entre aquelas crianças cujas mães possuíam de cinco a oito anos de estudo.

O consumo frequente de verduras de folhas e legumes foi mais prevalente entre as meninas, entre as crianças residentes na zona urbana e entre aquelas cujas mães possuíam 12 ou mais anos de estudo. Além disso, maior prevalência de consumo de verduras ocorreu na região Sul e de legumes na região Centro-oeste.

Quanto às frutas e ao suco natural, observou-se maior prevalência de consumo frequente

Tabela 2. Distribuição da frequência de consumo semanal de alimentos marcadores de alimentação saudável e não saudável entre crianças de dois a cinco anos de idade. Pesquisa Nacional de Demografia e Saúde (PNDS), 2006. $(\mathrm{n}=3.083)$.

\begin{tabular}{|c|c|c|c|c|c|}
\hline \multirow[b]{2}{*}{ Alimento } & \multicolumn{5}{|c|}{ Frequência de consumo (\%) } \\
\hline & Não comeu & 1 dia & $2-3$ dias & 4-6 dias & Diariamente \\
\hline \multicolumn{6}{|c|}{ Marcadores de alimentação saudável } \\
\hline Feijão & 6,1 & 4,4 & 13,5 & 7,8 & 68,2 \\
\hline Verduras de folhas & 49,6 & 12,2 & 18,2 & 6,2 & 13,8 \\
\hline Legumes $^{\mathrm{b}}$ & 25,9 & 12,5 & 26,8 & 15,3 & 19,5 \\
\hline Frutas & 10,4 & 7,2 & 24,5 & 14,1 & 43,8 \\
\hline Suco natural & 24,6 & 9,3 & 22,4 & 13,8 & 29,9 \\
\hline \multicolumn{6}{|c|}{ Marcadores de alimentação não saudável } \\
\hline Frituras & 40,5 & 23,0 & 23,0 & 7,3 & 6,2 \\
\hline Doces & 21,1 & 14,7 & 24,8 & 13,9 & 25,5 \\
\hline Biscoitos ou bolachas & 7,6 & 7,3 & 19,7 & 18,0 & 47,4 \\
\hline Salgadinhos em pacote & 38,0 & 23,7 & 20,8 & 8,0 & 9,5 \\
\hline Refrigerantes $^{c}$ & 18,0 & 17,4 & 27,4 & 11,9 & 25,3 \\
\hline
\end{tabular}

Percentual máximo de observações desconhecidas: $7,2 \%(\mathrm{n}=221)$ para a variável verduras de folhas. ${ }^{\text {a }}$ incluindo mandioquinha (batata- baroa), mandioca (macaxeira, aipim), cará, inhame e abóbora (jerimum). ${ }^{\mathrm{b}}$ incluindo tomate, cenoura, berinjela, beterraba, chuchu e abobrinha. ${ }^{~}$ incluindo sucos artificiais. 
Tabela 3. Consumo frequente de alimentos marcadores de alimentação saudável entre crianças de dois a cinco anos de idade, segundo características sociodemográficas. Pesquisa Nacional de Demografia e Saúde (PNDS), 2006.

\begin{tabular}{|c|c|c|c|c|c|}
\hline \multirow{2}{*}{ Variáveis } & \multicolumn{5}{|c|}{$\%($ IC95\%) } \\
\hline & Feijão & $\begin{array}{l}\text { Verduras de } \\
\text { folhas }\end{array}$ & Legumes $^{\mathrm{a}}$ & Frutas & Suco natural \\
\hline Idade da criança (meses) & $\mathrm{p}=0,1613$ & $\mathrm{p}=0,9924$ & $\mathrm{p}=0,5354$ & $\mathrm{p}=0,3657$ & $\mathrm{p}=0,3158$ \\
\hline $24-35$ & $73,2(68,3-78,1)$ & $19,8(15,7-23,8)$ & $36,7(31,6-41,8)$ & $57,2(51,8-62,5)$ & $44,9(39,5-50,2)$ \\
\hline $36-47$ & $79,5(75,3-83,8)$ & $20,1(15,9-24,2)$ & $35,2(29,8-40,5)$ & $55,6(50,1-61,0)$ & $40,3(35,1-45,6)$ \\
\hline $48-59$ & $75,1(70,0-80,1)$ & $20,1(16,0-24,3)$ & $32,5(27,1-37,8)$ & $61,0(55,5-66,5)$ & $46,1 \quad(40,1-52,1)$ \\
\hline Sexo & $\mathrm{p}=0,3622$ & $\mathrm{p}=0,0355$ & $\mathrm{p}=0,0173$ & $\mathrm{p}=0,7665$ & $\mathrm{p}=0,0186$ \\
\hline Meninos & $77,2(76,6-80,8)$ & $17,6(14,6-20,6)$ & $31,3(27,4-35,2)$ & $57,4(53,2-61,7)$ & $40,0(35,8-44,2)$ \\
\hline Meninas & $74,6(70,5-78,8)$ & $22,7(18,9-26,5)$ & $38,7(34,0-43,4)$ & $58,4(53,7-63,1)$ & $47,7(42,9-52,6)$ \\
\hline Macrorregião & $\mathrm{p}<0,001$ & $\mathrm{p}<0,001$ & $\mathrm{p}=0,0379$ & $\mathrm{p}=0,0001$ & $\mathrm{p}<0,001$ \\
\hline Norte & $55,4(50,3-60,5)$ & $18,7(14,7-22,7)$ & $28,7(24,1-33,3)$ & $45,3(40,3-50,4)$ & $36,1 \quad(31,2-40,9)$ \\
\hline Nordeste & $69,7(64,5-74,9)$ & $9,7(6,8-12,6)$ & $30,0(24,3-35,5)$ & $67,5(62,2-72,7)$ & $58,9(53,2-64,6)$ \\
\hline Sudeste & $83,0(77,8-88,1)$ & $23,9(19,0-28,7)$ & $7,4(31,6-43,2)$ & $54,6(48,4-60,7)$ & $40,0(33,9-46,2)$ \\
\hline Sul & $74,5(69,3-79,8)$ & $26,2(21,2-31,2)$ & $37,9(32,4-43,5)$ & $58,5(52,7-64,3)$ & $31,3(26,0-36,6)$ \\
\hline Centro-oeste & $88,5(84,7-92,3)$ & $25,8(20,4-31,1)$ & $40,2(34,5-45,9)$ & $56,6(50,9-62,3)$ & $40,3(34,7-45,9)$ \\
\hline Zona de moradia & $p=0,1516$ & $\mathrm{p}<0,001$ & $\mathrm{p}=0,0059$ & $\mathrm{p}=0,2958$ & $\mathrm{p}=0,8286$ \\
\hline Urbana & $76,8(73,6-80,0)$ & $21,9(19,1-24,8)$ & $37,0(33,4-40,5)$ & $58,6(54,9-62,3)$ & $43,9(40,2-47,6)$ \\
\hline Rural & $72,5(67,4-77,6)$ & $12,2(9,4-15,1)$ & $26,3(20,1-32,5)$ & $54,9(49,1-60,8)$ & $43,1(36,8-49,4)$ \\
\hline Idade materna (anos) & $\mathrm{p}=0,7052$ & $\mathrm{p}=0,1915$ & $\mathrm{p}=0,3980$ & $p=0,0216$ & $\mathrm{p}=0,5375$ \\
\hline$<20$ & $72,4(59,8-85,0)$ & $14,5(6,1-23,0)$ & $27,1(14,6-39,6)$ & $44,7(30,5-58,9)$ & $39,5(25,3-53,7)$ \\
\hline $20-29$ & $75,0(71,4-78,6)$ & $18,5(15,5-21,5)$ & $33,6(29,7-37,5)$ & $55,3(51,2-59,4)$ & $42,2(38,1-46,3)$ \\
\hline $30-39$ & $78,0(72,9-83,0)$ & $23,5(18,6-28,3)$ & $38,1(32,3-43,9)$ & $63,9(58,3-69,5)$ & $47,2(41,0-53,3)$ \\
\hline $40-49$ & $77,6(68,1-87,0)$ & $20,3(11,8-28,8)$ & $35,3(23,6-46,9)$ & $61,2(49,6-72,7)$ & $43,6(31,4-55,8)$ \\
\hline Escolaridade materna (anos) & $\mathrm{p}=0,0321$ & $\mathrm{p}<0,001^{\mathrm{b}}$ & $\mathrm{p}<0,001^{\mathrm{b}}$ & $\mathrm{p}=0,0003^{\mathrm{b}}$ & $\mathrm{p}=0,0004^{\mathrm{b}}$ \\
\hline $0-4$ & $76,3(70,9-81,5)$ & $11,3(8,2-14,3)$ & $21,5(16,6-26,5)$ & $47,8(41,3-54,3)$ & $36,7(30,3-43,2)$ \\
\hline $5-8$ & $79,8(75,6-84,1)$ & $17,1(13,2-21,0)$ & $29,5(24,5-34,6)$ & $57,5(52,1-62,9)$ & $37,6(32,2-43,0)$ \\
\hline $9-11$ & $69,6(63,9-75,4)$ & $24,3(19,4-29,2)$ & $43,6(37,9-49,3)$ & $61,9(56,1-76,6)$ & $47,4(41,7-53,2)$ \\
\hline 12 ou mais & $75,0(63,2-86,9)$ & $36,3(21,6-50,9)$ & $48,7(31,7-65,6)$ & $77,3(66,4-88,3)$ & $64,1(49,7-78,6)$ \\
\hline
\end{tabular}

Percentual máximo de observações desconhecidas: $17,2 \%(\mathrm{n}=531)$ para a variável escolaridade materna. ${ }^{a}$ incluindo tomate, cenoura, berinjela, beterraba, chuchu e abobrinha; ${ }^{\mathrm{b}}$ Teste de Wald para tendência linear. Valor-p estimado pelo teste Qui-quadrado de heterogeneidade

na região Nordeste e entre as crianças cujas mães possuíam maior escolaridade. O consumo de frutas também se mostrou mais prevalente entre as crianças cujas mães tinham de 30 a 39 anos de idade, e o consumo de suco de frutas foi mais prevalente entre as meninas.

Quando analisados os marcadores de alimentação não saudável segundo variáveis sociodemográficas (Tabela 4), verificou-se maior prevalência de consumo frequente de frituras na zona urbana e entre as crianças cujas mães possuíam menos de 20 anos de idade. Com relação aos doces, observou-se maior prevalência de consumo frequente na região Sul e na zona urbana.

O consumo frequente de biscoitos ou bolachas e salgadinhos em pacote foi registrado com maior prevalência na região Nordeste, sendo o consumo de biscoitos mais prevalente entre os meninos e entre as crianças cujas mães possuíam de 9 a 11 anos de estudo, e o consumo de salgadinhos entre as crianças cujas mães possuíam de 20 a 29 anos de idade.

Já em relação ao consumo frequente de refrigerantes e sucos artificiais, maiores prevalências foram observadas entre as crianças residentes na região Sul, na zona urbana e cujas mães possuíam de cinco a oito anos de estudo.

\section{Discussão}

Os resultados mostraram que a maioria das crianças de dois a cinco anos de idade, residentes nas diferentes regiões brasileiras, consome vegetais de folhas e legumes em uma frequência inferior a quatro dias na semana. Além disso, um grande número de crianças havia consumido alimentos marcadores de alimentação não saudá- 
Tabela 4. Consumo frequente de alimentos marcadores de alimentação não saudável entre crianças de dois a cinco anos de idade, segundo características sociodemográficas. Pesquisa Nacional de Demografia e Saúde (PNDS), 2006.

\begin{tabular}{|c|c|c|c|c|c|}
\hline \multirow{2}{*}{ Variáveis } & \multicolumn{5}{|c|}{$\%($ IC95\%) } \\
\hline & Frituras & Doce & $\begin{array}{l}\text { Biscoitos ou } \\
\text { bolachas }\end{array}$ & $\begin{array}{l}\text { Salgadinhos em } \\
\text { pacote }\end{array}$ & Refrigerantes $^{a}$ \\
\hline Idade da criança (meses) & $\mathrm{p}=0,6397$ & $\mathrm{p}=0,7485$ & $\mathrm{p}=0,3642$ & $\mathrm{p}=0,9320$ & $\mathrm{p}=0,2348$ \\
\hline $24-35$ & $13,7(9,6-17,8)$ & $41,1(35,7-46,6)$ & $67,4(62,7-72,1)$ & $17,7(13,4-21,9)$ & $33,7(28,5-39,0)$ \\
\hline $36-47$ & $14,6(10,7-18,6)$ & $38,6(33,2-44,0)$ & $66,3(61,1-71,5)$ & $16,8(13,1-20,6)$ & $40,3(34,9-45,7)$ \\
\hline $48-59$ & $12,1(9,0-15,3)$ & $38,4(32,9-44,0)$ & $62,2(56,3-68,2)$ & $17,9(13,4-22,4)$ & $37,6(32,0-43,1)$ \\
\hline Sexo & $\mathrm{p}=0,5881$ & $\mathrm{p}=0,9671$ & $\mathrm{p}=0,0442$ & $\mathrm{p}=0,5502$ & $\mathrm{p}=0,2252$ \\
\hline Meninos & $14,1(10,9-17,2)$ & $39,3(35,1-43,6)$ & $68,3(64,5-72,2)$ & $18,1(14,9-21,4)$ & $39,0(34,8-43,3)$ \\
\hline Meninas & $12,9(9,9-15,9)$ & $39,5(34,8-44,2)$ & $62,1(57,3-66,8)$ & $16,7(13,1-20,2)$ & $35,2(30,6-39,7)$ \\
\hline Macrorregião & $\mathrm{p}=0,8244$ & $\mathrm{p}<0,001$ & $\mathrm{p}=0,0001$ & $\mathrm{p}=0,0016$ & $\mathrm{p}<0,001$ \\
\hline Norte & $12,0(8,8-15,2)$ & $27,7(23,2-32,2)$ & $50,8(45,6-55,9)$ & $10,1(6,8-13,4)$ & $29,6(24,9-34,3)$ \\
\hline Nordeste & $13,1(8,6-17,6)$ & $34,2(28,3-40,2)$ & $73,8(69,1-78,5)$ & $23,8(19,1-28,5)$ & $22,6(17,2-28,0)$ \\
\hline Sudeste & $14,3(10,3-18,2)$ & $39,3(33,3-45,3)$ & $64,3(58,2-70,3)$ & $14,7(10,2-19,2)$ & $42,9(36,9-48,9)$ \\
\hline Sul & $14,2(9,7-18,7)$ & $57,6(52,0-63,1)$ & $62,2(56,6-67,8)$ & $18,7(14,1-23,2)$ & $55,1(49,4-60,7)$ \\
\hline Centro-oeste & $11,5(8,1-15,0)$ & $42,5(36,8-48,2)$ & $64,9(59,2-70,5)$ & $17,2(13,3-21,1)$ & $37,3(31,5-43,1)$ \\
\hline Zona de moradia & $\mathrm{p}=0,0477$ & $\mathrm{p}=0,0036$ & $\mathrm{p}=0,3294$ & $\mathrm{p}=0,2046$ & $\mathrm{p}<0,001$ \\
\hline Urbana & $14,4(11,8-17,0)$ & $41,6(37,9-45,3)$ & $66,0(62,4-69,6)$ & $18,2(15,4-21,0)$ & $40,4(36,8-44,0)$ \\
\hline Rural & $10,0(6,8-13,2)$ & $30,6(24,5-36,7)$ & $62,8(57,5-68,1)$ & $14,7(10,4-19,0)$ & $24,6(18,9-30,4)$ \\
\hline Idade materna (anos) & $\mathrm{p}=0,0127$ & $\mathrm{p}=0,8564$ & $\mathrm{p}=0,2783$ & $\mathrm{p}=0,0281$ & $\mathrm{p}=0,2871$ \\
\hline$<20$ & $19,9(5,6-34,2)$ & $39,6(25,3-53,9)$ & $63,4(50,5-76,3)$ & $17,9(8,1-27,6)$ & $34,1(19,7-48,5)$ \\
\hline $20-29$ & $15,9(12,8-19,0)$ & $39,0(34,9-43,1)$ & $67,7(63,9-71,5)$ & $20,3(16,8-23,7)$ & $39,0(34,9-43,0)$ \\
\hline $30-39$ & $9,6(6,7-12,6)$ & $38,9(33,1-44,7)$ & $63,2(57,0-69,3)$ & $13,4(10,0-16,90)$ & $33,2(27,6-38,7)$ \\
\hline $40-49$ & $6,6(2,7-10,4)$ & $44,6(32,2-56,9)$ & $57,6(46,2-69,0)$ & $12,0(4,6-19,4)$ & $43,2(30,9-55,5)$ \\
\hline Escolaridade materna (anos) & $\mathrm{p}=0,2397$ & $\mathrm{p}=0,1376$ & $\mathrm{p}=0,0496$ & $\mathrm{p}=0,3286$ & $\mathrm{p}=0,0081$ \\
\hline $0-4$ & $11,0(6,2-15,7)$ & $32,2(25,9-38,5)$ & $58,1(52,1-64,1)$ & $21,3(15,1-27,4)$ & $30,6(24,3-36,9)$ \\
\hline $5-8$ & $16,6(12,5-20,7)$ & $43,1(37,5-48,7)$ & $63,9(58,7-69,1)$ & $17,5(13,9-21,2)$ & $43,3(37,7-48,9)$ \\
\hline $9-11$ & $12,0(8,2-15,8)$ & $39,7(34,0-45,4)$ & $69,5(64,1-74,9)$ & $15,6(11,2-20,0)$ & $37,7(32,2-43,2)$ \\
\hline 12 ou mais & $16,3(6,2-26,5)$ & $45,2(28,9-61,4)$ & $52,8(35,0-70,6)$ & $12,3(2,7-21,9)$ & $25,5(13,8-37,1)$ \\
\hline
\end{tabular}

Percentual máximo de observações desconhecidas:17,1\% ( $\mathrm{n}=526)$ para a variável escolaridade materna. ${ }^{a}$ incluindo sucos artificiais. Valor-p estimado pelo teste Qui-quadrado de heterogeneidade.

vel, como frituras, doces e refrigerantes em pelo menos uma vez nos últimos sete dias.

Estes achados demonstram que o consumo alimentar de crianças nessa faixa etária é uma questão a ser trabalhada. A ingestão insuficiente de alimentos como legumes e vegetais compromete o aporte de micronutrientes, podendo influenciar negativamente na reação do sistema imunológico das crianças frente a infecções ${ }^{4,5}$. É possível que esse comportamento seja reflexo da alimentação da família, pois se sabe que o consumo de frutas, legumes e verduras entre adultos está aquém do recomendado ${ }^{16}$ e que as crianças tendem a consumir o que as pessoas que as cercam consomem ${ }^{17-19}$.

O consumo frequente de refrigerantes e sucos artificiais é outro aspecto que merece especial atenção, tendo em vista que o consumo dessas bebidas está associado à redução da ingestão de leite e suco de frutas naturais ${ }^{20}$. Além disso, o aumento do consumo de bebidas açucaradas está associado ao aumento de ingestão calórica, ganho de peso e suas consequências ${ }^{21,22}$. No mesmo sentido, o maior consumo de frituras, doces e biscoitos, sobretudo os recheados, contribui para o aumento da densidade energética das refeições aumentando o risco de obesidade ${ }^{5}$.

O consumo elevado de alimentos industrializados pode ser reflexo do aumento da disponibilidade domiciliar desses alimentos que, segundo a Pesquisa de Orçamentos Familiares, aumentou em $400 \%$ tanto para biscoitos ou bolachas como para refrigerantes no período de 1974-1975 a 2002-2003 23 . Dados da Pesquisa Nacional por Amostras de Domicílios mostram que houve aumento da renda per capta da população brasi- 
leira com incremento da Classe C, principalmente entre 2003 e $2009^{24}$, o que pode ter favorecido a aquisição desses gêneros. Além da melhoria na renda da população, estes alimentos passaram a ser comercializados com preços mais acessíveis às classes D e E. Estas mudanças podem, em grande parte, ser responsáveis pelo aumento da aquisição e do consumo desses alimentos.

Ao analisar o consumo frequente dos alimentos marcadores de alimentação saudável e não saudável segundo as variáveis sociodemográficas, não se observou diferença no consumo desses alimentos conforme a idade das crianças. Cooke et al. ${ }^{25}$ também não encontraram relação entre o consumo de frutas e a idade da criança, mas observaram que as crianças mais velhas consumiam mais frequentemente vegetais em relação às mais jovens. Diferentemente, o estudo de Farias Júnior e Osório ${ }^{26}$, com crianças de zero a cinco anos, mostrou que o consumo de açúcar, amido de milho e alimentos à base de cereal diminuiu com o avanço da idade, ao passo que o consumo de gordura, feijão e carne aumentou conforme maior a idade das crianças.

Quanto ao sexo, houve maior consumo de alimentos marcadores de alimentação saudável (verduras de folhas, legumes e suco natural) entre as meninas. Em estudo realizado por Glynn et al. ${ }^{27}$, também observou-se que as meninas consumiam mais frutas e vegetais do que os meninos. Esse dado também foi confirmado por Northstone e Emmett ${ }^{28}$, que observaram um maior consumo de uma dieta "tradicional", constituída principalmente de carnes, frutas e vegetais entre as meninas. Esta diferença do consumo alimentar entre os sexos pode ser atribuída a diferenças na socialização alimentar entre as meninas e os meninos ${ }^{17}$. Segundo Oliveira et al. ${ }^{29}$, o contexto social no momento das refeições influencia o interesse intrínseco pelo tipo de alimento a ele associado, pois este passaria a representar o contexto emocional em que é habitualmente consumido. Porém, no presente estudo não é possível afirmar que as diferenças entre meninos e meninas se devem à socialização alimentar, pois a pesquisa original da PNDS não oferece ferramentas para a análise desta questão.

As crianças residentes na zona urbana apresentaram as maiores prevalências de consumo de verduras em folhas, legumes, frituras, doces e refrigerantes. Fidelis e Osório ${ }^{30}$ observaram que houve menor prevalência de inadequação de consumo de vitaminas e minerais na zona urbana de
Pernambuco em crianças menores de cinco anos. Segundo Farias Júnior e Osório ${ }^{26}$, a diversidade de alimentos em uma região mais desenvolvida (metropolitana) em relação à outra menos desenvolvida (interior urbano e rural) pode influenciar o consumo alimentar. Quanto às macrorregiões, a região Sul apresentou maior prevalência de consumo frequente de verduras de folhas, doces e refrigerantes, confirmando os achados da POF 2008-2009, a qual relatou que as maiores prevalências de aquisição desses produtos foram observadas também para a macrorregião Sul ${ }^{9}$.

O presente estudo corrobora achados de outros autores ${ }^{31-34}$ ao observar que as crianças cujas mães possuíam menor idade e escolaridade apresentavam uma alimentação de menor qualidade. Provavelmente, porque mães com mais anos de estudo e idade têm maior possibilidade de discernir entre o que é considerado de fato alimento saudável ou não ${ }^{26,33,35}$. Além disso, soma-se o fato de a escolaridade estar bastante associada à classe social. Assim, o consumo de alimentos mais saudáveis, como frutas e verduras estaria intimamente relacionado ao aumento da renda, tendência essa, confirmada pelas Pesquisas de Orçamento Familiar ${ }^{9,23}$.

Estudos envolvendo as práticas alimentares de crianças são escassos na literatura científica e os poucos trabalhos que descrevem hábitos alimentares nesse grupo etário geralmente adotam esquemas de coleta dos dados dietéticos e classificação das frequências de consumo diferentes, limitando muitas vezes, as comparações entre os estudos. Além disso, são poucos os estudos que analisam o consumo alimentar por meio de marcadores alimentares.

\section{Conclusão}

Tendo em vista os dados observados neste estudo, reforça-se a importância de intensificar o esclarecimento sobre os benefícios de uma alimentação saudável na infância, em especial do consumo de frutas, verduras e legumes. Além disso, é importante alertar sobre as consequências negativas do consumo de doces e refrigerantes nessa fase da vida. A promoção do consumo de frutas e suco de frutas em substituição ao de doces e refrigerantes também deve ser adotado. Aliada a essas ações, no processo de educação alimentar, torna-se necessário rever a abordagem empregada para estimular a alimentação saudável. 


\section{Colaboradores}

MFA Vieira e LC Muniz orientaram a escrita, análise e interpretação dos dados, além de revisarem o artigo. MN Alves realizou a análise dos dados e redigiu o artigo.

\section{Referências}

1. Tuma RCFB, Costa THM, Schmitz BAS. Avaliação antropométrica e dietética de pré-escolares em três creches de Brasília, Distrito Federal. Rev. Bras. Saúde Matern. Infant. 2005; 5(Supl. 4):419-428.

2. Menezes RCE, Osório MM. Consumo energéticoprotéico e estado nutricional de crianças menores de cinco anos, no estado de Pernambuco, Brasil. Rev. Nutr. 2007; 20(Supl. 4):337-347.

3. Vitolo MR. Nutrição da gestação ao envelhecimento. Rio de Janeiro: Reichmann \& Affonso Editores; 2008.

4. Macedo EMC, Amorim MAF, Silva ACS, Castro CMMB. Efeitos da deficiência de cobre, zinco e magnésio sobre o sistema imune de crianças com desnutrição grave. Rev Paul Pediatr 2010; 28(Supl. 3):329-336

5. Cagliari MPP, Paiva AA, Queiroz D, Araujo ES. Consumo alimentar, antropometria e morbidade em pré-escolares de creche públicas de Campina Grande, Paraíba. Nutrire 2009; 34(Supl. 1):29-43.

6. Victora CG, Adair L, Fall C, Hallal PC, Martorell R, Richter L, Sachdev HS. Maternal and child undernutrition: consequences for adult health and human capital. The Lancet 2008; 371(9609):340-357.

7. Huybrechts I, Matthys C, Vereecken C, Maes L, Temme EHM, Oyen HV, Backer G, Henauw S. Food Intakes by Preschool Children in Flanders Compared with Dietary Guidelines. Int J Environ Res Public Health 2008; 5(4):243-257.

8. Levy-Costa RB, Sichieri R, Pontes NS, Monteiro CA. Disponibilidade de alimentos no Brasil: distribuição e evolução (1974-2003). Rev Saude Publica 2005; 39(Supl. 4):530-540.

9. Instituto Brasileiro de Geografia e Estatística (IBGE). Pesquisa de Orçamentos Familiares 2008-2009. Avaliação nutricional da disponibilidade domiciliar de alimentos no Brasil. Rio de Janeiro, 2010. [documento na Internet]. [acessado 2011 jun 13]. Disponível em: http://www.ibge.gov.br.

10. Cavalcante AAM, Priore SE, Franceschini SCC. Estudos de consumo alimentar: aspectos metodológicos gerais e o seu emprego na avaliação de criancas e adolescentes. Rev. Bras. Saúde Matern. Infant. 2004; 4(Supl. 3):229-240.

11. Brasil. Ministério da Saúde (MS). Pesquisa Nacional de Demografia e Saúde da criança e da Mulher (PNDS-2006). 2008. [documento na Internet]. [acessado 2011 jun 13]. Disponível em: http://www.saude. gov.br.

12. World Health Organization (WHO). Global strategy on diet, physical activity and health: fifty-seventh world health assembly. Genebra: WHO; 2004. 
13. Ceriello A, Quagliaro L, Piconi L, Assaloni R, Da Ros R, Maier A, Esposito K, Giugliano D. Effect of postprandial hypertrigliceridemia and hyperglicemia on circulating adhesion molecules and oxidative stress generation and the possible role of simvastatin treatment. Diabetes 2004; 53(Supl. 3):701-710.

14. Lopes-Garcia E, Schulze MB, Fung TT, Meigs JB, Rifai N, Manson JE, Hu FB. Major dietary patterns are related to plasma concentrations for markers of inflammation and endothelial dysfunction. Am J Clin Nutr 2004; 80(Supl. 4):1029-1035.

15. Levy RB, Castro IRR, Cardoso LO, Tavares LF, Sardinha LMV, Gomes FS, Costa AWN. Consumo e comportamento alimentar entre adolescentes brasileiros: Pesquisa Nacional de Saúde do Escolar (PeNSE), 2009. Cien Saude Colet 2010; 15(Supl. 2):3085-3097.

16. Jaime PC, Monteiro CA. Fruit and vegetable intake by Brazilian adults, 2003. Cad Saude Publica. 2005; 21(Supl. 1):19-24.

17. Ramos M, Stein LM. Desenvolvimento do comportamento alimentar infantil. J. Pediatr. 2000; 76(Supl. 3):229-237.

18. Pontes TE, Costa TF, Marum ABRF, Brasil ALD, Taddei JA AC. Orientação Nutricional de crianças e adolescentes e os novos padrões de consumo: propagandas, embalagens e rótulos. Rev Paul Pediatr 2009; 27(Supl. 1):99-105.

19. Mondini L, Levy RB, Saldiva SRDM, Venâncio SI, Aguiar JA, Stefanini MLR. Prevalência de sobrepeso e fatores associados em crianças ingressantes no ensino fundamental em um município da região metropolitana de São Paulo, Brasil. Cad Saude Publica 2007; 23(8):1825-1834.

20. Kaur H, Hyder ML, Poston WS. Childhood overweight: an expanding problem. Treat Endocrinol 2003; 2(6):375-388

21. Malik VS, Schulze MB, Hu FB. Intake of sugarsweetened beverages and weight gain: a systematic review. Am J Clin Nutr 2006; 84(2):274-288.

22. Vartanian LR, Schwartz MB, Brownell KD. Effects of Soft Drink Consumption on Nutrition and Health: A Systematic Review and Meta-Analysis. Am J Public Health 2007; 97(4):667-675.

23. Instituto Brasileiro de Geografia e estatística (IBGE). Pesquisa de Orçamentos Familiares (POF) 2002-2003. Análise da disponibilidade domiciliar de alimentos e do estado nutricional no Brasil. 2004. [documento na Internet]. [acessado 2012 jul 5]. Disponível em: http://www.ibge.gov.br.

24. Fundação Getúlio Vargas (FGV). A nova classe média: O lado brilhante dos pobres. 2010. [documento na Internet]. [acessado 2012 jun 29]. Disponível em: http://www.cps.fgv.br
25. Cooke LJ, Wardle J, Gibson EL, Sapochnik M, Sheiham A, Lawson M. Demographic, familial and trait predictors of fruit and vegetable consumption by pre-school children. Public Health Nutrition. 2003; 7(Supl.2):295-302.

26. Farias Júnior G, Osório MM. Padrão alimentar de crianças menores de cinco anos. Rev. Nutr. 2005; 18(Supl. 6):793-802.

27. Glynn L, Emmett P, Rogers I, The ALSPAC Study Team. Food and nutrient intakes of a population sample of 7-year-old children in the south-west of England in 1999/2000 - what difference does gender make? J Hum Nutr Dietet 2005; 18(1):7-19.

28. Northstone K, Emmett P. Multivariate analysis of diet in children at four and seven years of age and associations with socio-demographic characteristics. Eur J Clin Nutr 2005; 59(6):751-760.

29. Oliveira EA, Frizzo GB, Marin AH. Atitudes maternas diferenciais para com meninos e meninas de quatro e cinco anos. Psicologia: Reflexão e Crítica. 2000; 13(Supl. 3):363-371.

30. Fidelis CMF, Osório MM. Consumo alimentar de macro e micronutrientes de crianças menores de cinco anos no Estado de Pernambuco, Brasil. Rev. Bras. Saúde Matern. Infant. 2007; 7(Supl. 1):63-74.

31. Viana V, Santos PL, Guimarães MJ. Comportamento e hábitos alimentares em crianças e jovens: uma revisão da literatura. Psicologia, saúde \& doenças 2008; 9(Supl. 2):209-231.

32. Navia B, Ortega RM, Rodriguez-Rodriguez E, Aparicio A, Perea JM. La edad de la madre como condicionante del consumo de alimentos y la ingesta de energía y nutrientes de sus hijos en edad preescolar. Nutr Hosp 2009; 24(4):452-458.

33. Molina M CB, Lopéz PM, Faria CP, Cade NV, Zandonade E. Preditores socioeconômicos da qualidade da alimentação de crianças. Rev Saude Publica 2010; 44(Supl.5):785-792.

34. Dubois L, Farmer A, Girard M, Burnier D, Porcherie M. Demographic and socio-economic factors related to food intake and adherence to nutritional recommendations in a cohort of pre-school children. Public Health Nutrition.2011; 14(Supl. 6):1096-1104.

35. McDermott AJ, Stephens MB. Cost of Eating: whole foods versus convenience foods in a low-income model. Fam Med. 2010; 42(Supl. 4):280-284.

Artigo apresentado em 12/07/2012

Aprovado em 30/09/2012

Versão final apresentada em 17/10/2012 
